\title{
Protective effect of hydrogen sulphide against myocardial hypertrophy in mice
}

\author{
Mingjing Shao ${ }^{1, *}$, Chuanjun Zhuo ${ }^{2,3,4, *}$, Ronghuan Jiang ${ }^{5}$, Guangdong Chen ${ }^{2}$, Jianmin \\ Shan', Jing Ping ${ }^{2}$, Hongjun Tian ${ }^{3}$, Lina Wang ${ }^{3}$, Chongguang Lin ${ }^{2}$ and Lirong $\mathrm{Hu}^{2}$ \\ ${ }^{1}$ National Integrated Traditional and Western Medicine Center for Cardiovascular Disease, China-Japan Friendship Hospital, \\ Beijing, China \\ 2 Department of Psychological Medicine, Wenzhou Seventh People's Hospital, Wenzhou, China \\ ${ }^{3}$ Department of Psychological Medicine, Tianjin Anding Hospital, Tianjin, China \\ ${ }^{4}$ Department of Psychological Medicine, Tianjin Anning Hospital, Tianjin, China \\ ${ }^{5}$ Department of Psychological Medicine, Chinese People's Liberation Army General Hospital, Chinese People's Liberation \\ Army Medical School, Beijing, China \\ * These authors have contributed equally to this work
}

Correspondence to: Lirong Hu, email: cjzhuocambridge@vip.163.com

Keywords: hydrogen sulfide; cardiac hypertrophy; oxidative stress; Nrf2; Pathology Section

Received: December 12,2016 Accepted: February 20,2017 Published: February 28, 2017

\section{ABSTRACT}

Cardiac hypertrophy is a critical component of phenotype in the failing heart. Recently, increasing evidence has demonstrated that oxidative stress plays an important role in the pathogenesis of myocardial hypertrophy. In the present study, we generated a mouse model of transverse aortic constriction (TAC) to investigate whether hydrogen sulfide $\left(\mathrm{H}_{2} \mathrm{~S}\right)$ has protective effects against cardiac hypertrophy. Left ventricular structure was analyzed by two-dimensional echocardiography. Oxidative stress was evaluated by measuring malondialdehyde, superoxide dismutase, glutathione peroxidase and reactive oxygen specie in the myocardium. Angiotensin II (Ang-II) was used to induce cardiomyocyte hypertrophy. Neonatal rat cardiomyocytes pretreated with $\mathrm{H}_{2} \mathrm{~S}$ donor sodium hydrosulfide prior to Ang-II exposure were used to determine the involvement of Nrf2 and PI3K/Akt pathway in the antioxidant effects of $\mathrm{H}_{2} \mathrm{~S}$. Our findings showed that $\mathrm{H}_{2} \mathrm{~S}$ could protect against cardiac hypertrophy by attenuating oxidative stress. The antioxidant roles of $\mathrm{H}_{2} \mathrm{~S}$ in myocardial hypertrophy probably depend on the activation of PI3K/Akt signaling, which consequently increases Nrf2 activity and $\mathrm{HO}-1$ and GCLM expression. In summary, $\mathrm{H}_{2} \mathrm{~S}$ may exert antioxidant effect on cardiac hypertrophy via PI3K/Akt-dependent activation of Nrf2 pathway.

\section{INTRODUCTION}

Cardiac hypertrophy, which is characterized by myocardial fibrosis, capillary rarefaction, inflammatory reaction, and cellular dysfunction, consequently results in maladaptive ventricular remodeling and heart failure. The mechanical stress and neurohumoral stimulation are primary triggering events of myocardial hypertrophy. These events are associated with various cellular responses including gene transcription, protein translation, sarcomere assembly and cell metabolism, thereby leading to the progression of cardiac hypertrophy $[1,2]$. Under normal conditions, ventricular function is initially maintained by cardiac hypertrophy induced by pressure overload, and this is known as the adaptive phase. However, sustained pressure overload can promote the transition from the adaptive to maladaptive phase, which is characterized by left ventricular enlargement and dysfunction [3].

In recent years, hydrogen sulphide $\left(\mathrm{H}_{2} \mathrm{~S}\right)$ has been recognized as a new gaseous signaling molecule aside from nitric oxide and carbon monoxide. It has multiple physiological and pharmacological properties such as cardioprotection, vasorelaxation, antioxidant and antiinflammatory effects [4]. In the cardiovascular system, $\mathrm{H}_{2} \mathrm{~S}$ is produced in the myocardium and blood vessels from L-cysteine by cystathionine $\gamma$-lyase (CSE). Recently, 
there is growing evidence that $\mathrm{H}_{2} \mathrm{~S}$ plays important roles in the pathogenesis of various cardiovascular diseases [5, 6]. $\mathrm{H}_{2} \mathrm{~S}$ has been found to attenuate myocardial ischemiareperfusion injury by preservation of mitochondrial function and to reduce the mortality of heart failure induced by myocardial ischemia [7, 8]. In addition, $\mathrm{H}_{2} \mathrm{~S}$ has been shown to improve myocardial fibrosis by inhibiting oxidative stress, blocking TGF- $\beta 1 / \mathrm{Smad} 2$ signaling pathway, and reducing $\alpha$-SMA expression in cardiac fibroblasts [9]. In the present study, we generated a mouse model of transverse aortic constriction (TAC) to investigate whether $\mathrm{H}_{2} \mathrm{~S}$ has protective effect against cardiac hypertrophy.

\section{RESULTS}

$\mathrm{H}_{2} \mathrm{~S}$ levels in the myocardium were markedly lower in the TAC group than in the Sham group, while in the TAC+NaHS group, $\mathrm{H}_{2} \mathrm{~S}$ levels were significantly elevated compared with in the TAC group (Figure 1A). Moreover, the mRNA and protein expression of CSE was remarkably downregulated in the TAC group compared with in the Sham group (Figure 1B-1D).

The echocardiography was performed to evaluate cardiac hypertrophy. LVPWd, LVPWs, IVSd and IVSs were significantly increased in the TAC group compared
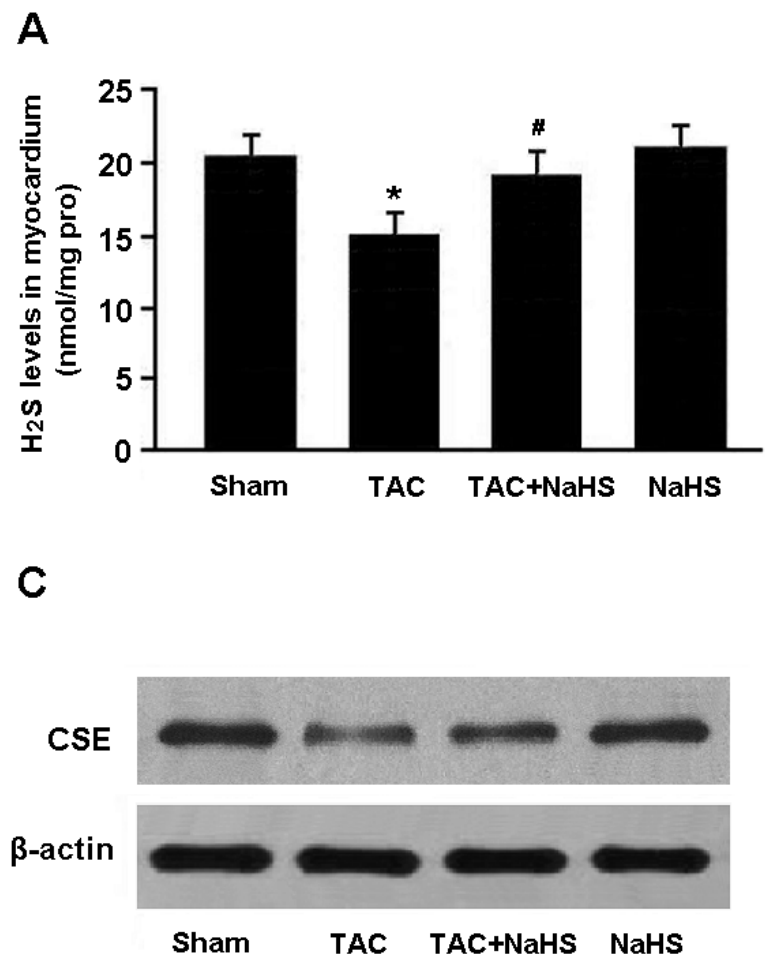

with in the Sham group, while in the TAC+NaHS group, these parameters were remarkably decreased compared with in the TAC group (Figure 2A-2D).

Left ventricular tissue was stained with $\mathrm{HE}$ and CSA was found to be significantly increased in the TAC mice and reduced after treatment with NaHS (Figure 3A, 3B). In addition, the expressions of ANF, BNP and $\beta-\mathrm{MHC}$, indicators of myocardial hypertrophy, were remarkably increased in the TAC group, while their expressions were markedly decreased following treatment with NaHS (Figure 3C-3E).

Oxidative stress was evaluated by detecting MDA, SOD, GSH-Px and ROS in the myocardium (Figure 4A4D). There were significant increase in MDA levels and decrease in SOD and GSH-Px activities in TAC mice, while NaHS treatment was found to reduce MDA levels and enhance SOD and GSH-Px activities. In addition, the ROS generation in myocardial tissue was remarkably elevated in the TAC group and reduced in the TAC+NaHS group.

As shown in Figure 5A, the Nrf2 protein accumulated in the myocardial cell nucleus and Nrf2-ARE binding activity was increased in the myocardium of TAC mice after treatment with NaHS. Moreover, the protein expressions of HO-1 and GCLM, downstream targets of Nrf2, were significantly upregulated in the TAC+NaHS group compared with in the TAC group (Figure 5B).

\section{B}
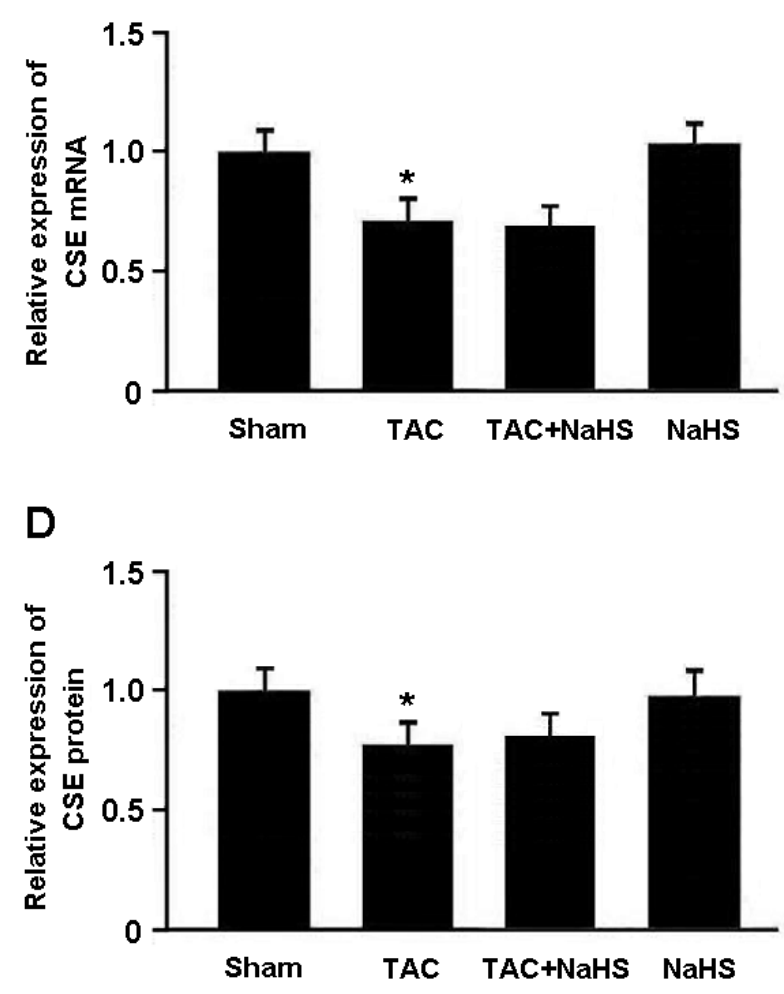

Figure 1: A. The $\mathrm{H}_{2} \mathrm{~S}$ levels in myocardial tissue were measured by the methylene blue method. B.-D. The mRNA and protein expression of cystathionine $\gamma$-lyase (CSE) was determined by real-time PCR and Western blotting. ${ }^{*} P<0.05$, vs. Sham; ${ }^{\#} P<0.05$, vs. TAC ( $n=5$ ). 
To determine whether $\mathrm{H}_{2} \mathrm{~S}$ attenuates Ang-IIinduced oxidative stress in the Nrf2-dependent manner, we transfected cardiomyocytes with Nrf2 siRNA and then subjected them to Ang-II. Our results indicated that Nrf2 siRNA-transfected cells that had been exposed to Ang-II and NaHS showed decreased nuclear expression of Nrf2 and elevated ROS generation compared with cells that had not been transfected with Nrf2 siRNA (Figure 6A, 6B).

To confirm whether $\mathrm{H}_{2} \mathrm{~S}$ regulates $\mathrm{Nrf} 2$ via $\mathrm{PI} 3 \mathrm{~K} /$ Akt-dependent pathway, we transfected cardiomyocytes with PI3K siRNA and then subjected them to Ang-II. Our results showed that PI3K siRNA-transfected cells exposed to Ang-II and NaHS exhibited reduced expression of phosphorylated Akt and nuclear Nrf2 and increased production of ROS compared with cells not transfected with PI3K siRNA (Figure 7A-7D).

\section{DISCUSSION}

In this study, we established a mouse model of cardiac hypertrophy using TAC method and found that NaHS treatment could significantly attenuate myocardial

A

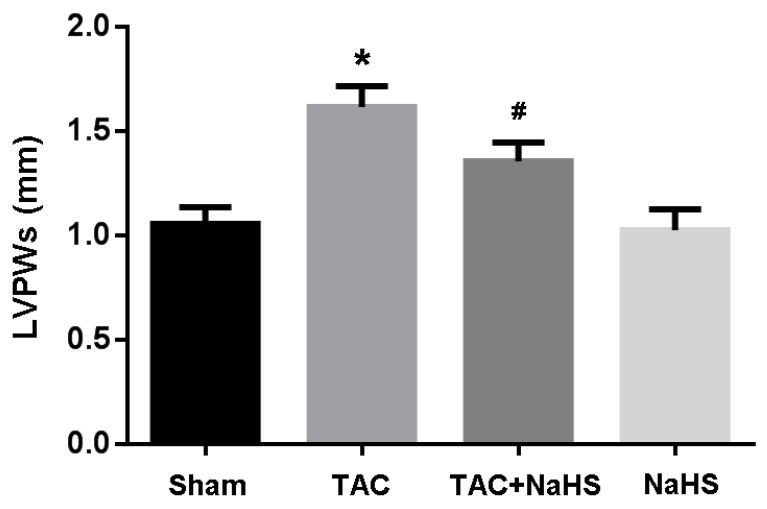

C

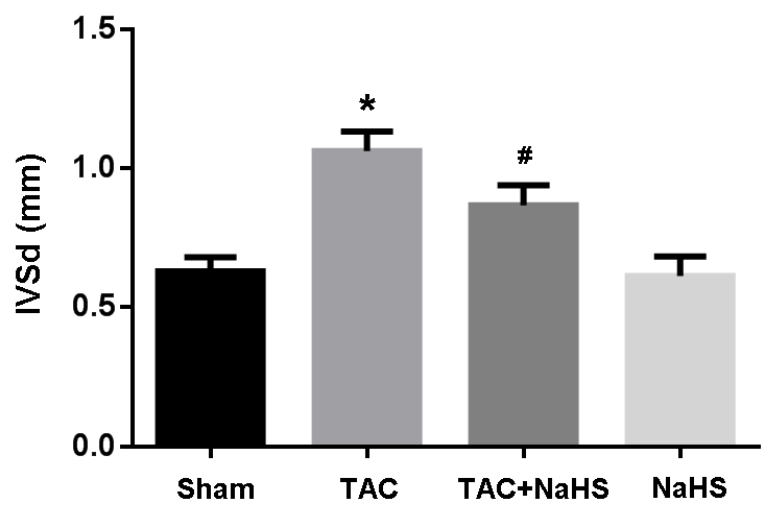

hypertrophy and regulate the expression of hypertrophyassociated genes in TAC mice. In addition, we also found that endogenous generation of $\mathrm{H}_{2} \mathrm{~S}$ and $\mathrm{CSE}$ expression were remarkably decreased in hypertrophic mice, while exogenous supplement of NaHS increased $\mathrm{H}_{2} \mathrm{~S}$ levels in the myocardial tissue.

Cardiac hypertrophy is a critical component of phenotype in the failing heart. In recent years, accumulating evidence has demonstrated that oxidative stress plays important roles in the pathogenesis of myocardial hypertrophy either in response to chronic pressure overload or neurohumoral stimuli. In cultured myocardial cells, hypertrophy induced by angiotensin II, endothelin 1 or norepinephrine was found to be associated with increased ROS generation [10]. The potential mechanisms involved include ROS-induced activation of mitogen activated protein kinases and nuclear factor$\kappa \mathrm{B}$. In pressure overload-mediated cardiac hypertrophy, NADPH oxidase existing in myocardial cells is the main source of ROS production and may result in various pathophysiological changes such as the activation of redox enzyme and progression to ventricular remodeling [11].

\section{B}

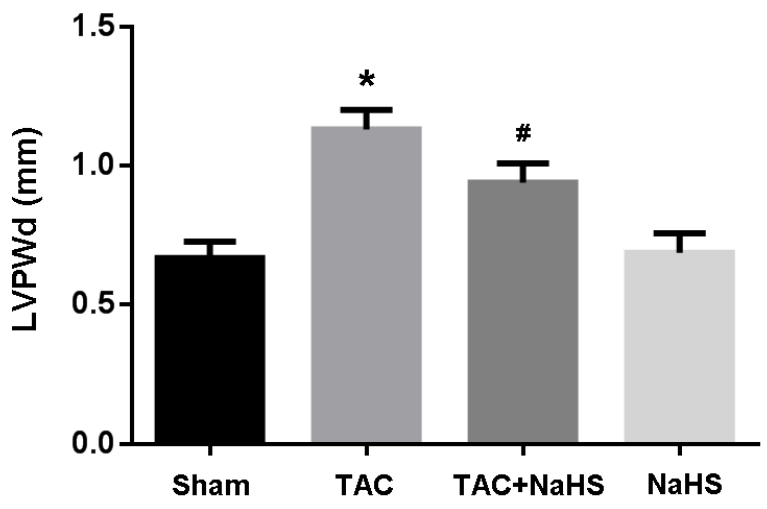

D

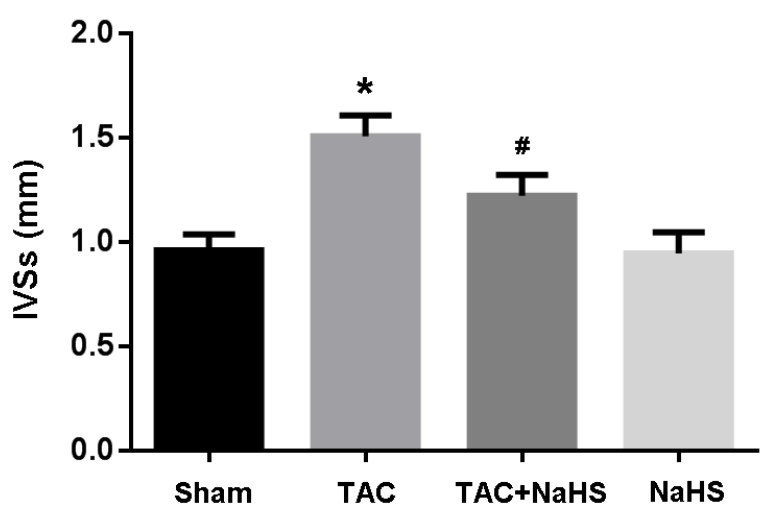

Figure 2: Echocardiographic evaluation of cardiac hypertrophy. A. Left ventricular posterior wall thickness at end-systole (LVPWs); B. Left ventricular posterior wall thickness at end-diastole (LVPWd); C. Interventricular septum thickness at end-diastole (IVSd); D. Interventricular septum thickness at end-systole (IVSs). ${ }^{*} P<0.05$, vs. Sham; ${ }^{\#} P<0.05$, vs. TAC $(n=5)$. 
In the present study, oxidative stress was evaluated by detecting MDA, SOD, GSH-Px and ROS in the myocardium. Our findings revealed that ROS generation was significantly increased in the mouse model of cardiac hypertrophy. In addition, $\mathrm{H}_{2} \mathrm{~S}$ was found to significantly attenuate oxidative stress in the myocardium of hypertrophic mice, which might be a critical protective mechanism against TAC-induced myocardial hypertrophy.

Nrf2 is an important regulator of endogenous antioxidant systems. Under normal conditions, Nrf2 locates in the cytoplasm and binds with Keap1 to mediate a rapid ubiquitination and degradation of $\mathrm{Nrf} 2$ by the proteasome. In response to oxidative stress, Nrf2 is released from Keap1 and transfers into the nucleus to correlate with ARE in the promoters of antioxidant enzyme regulatory genes [12]. In the present study, $\mathrm{H}_{2} \mathrm{~S}$ was found to enhance the binding activity of Nrf2ARE and increase the protein expression of antioxidant enzymes HO-1 and GCLM, which consequently augments the resistance to oxidative stress in the hypertrophic mice.

A

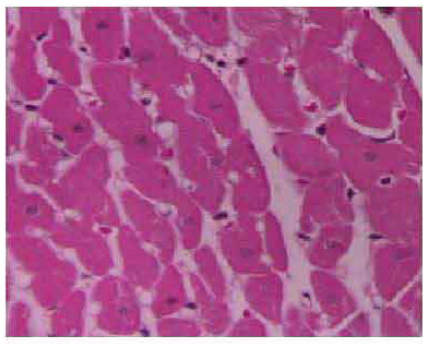

Sham

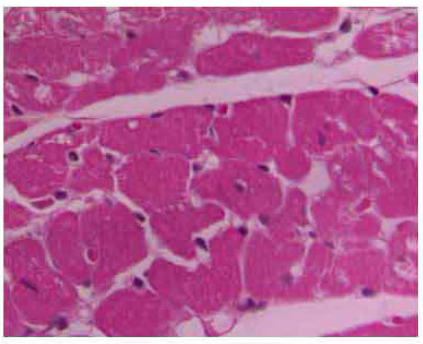

TAC

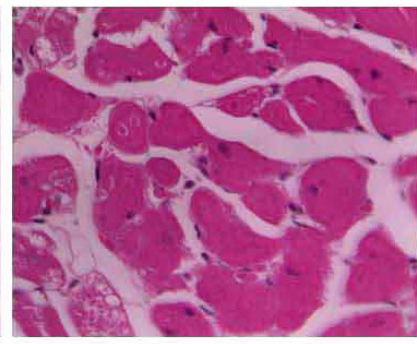

TAC+NaHS

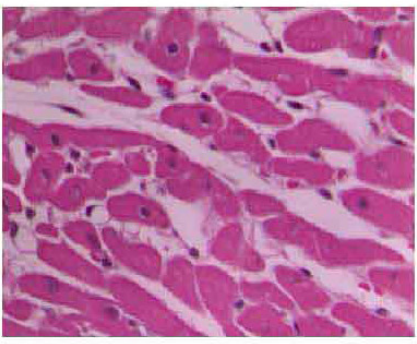

NaHS

\section{B}

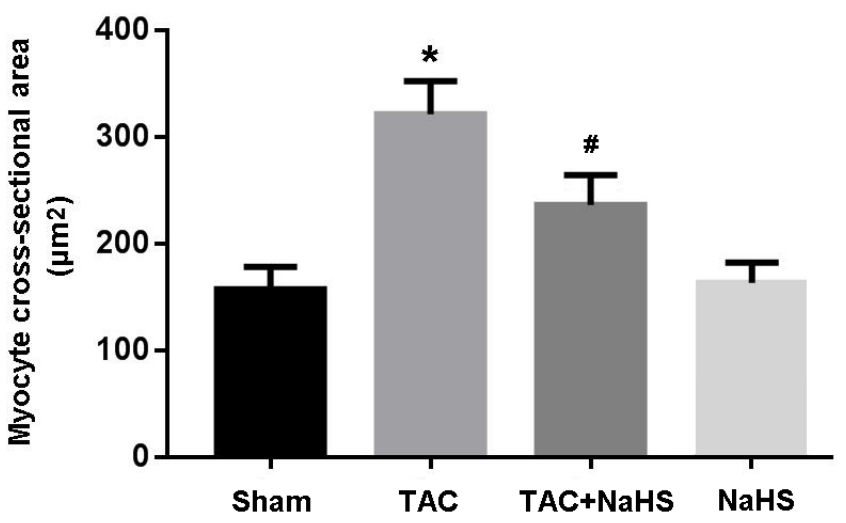

D

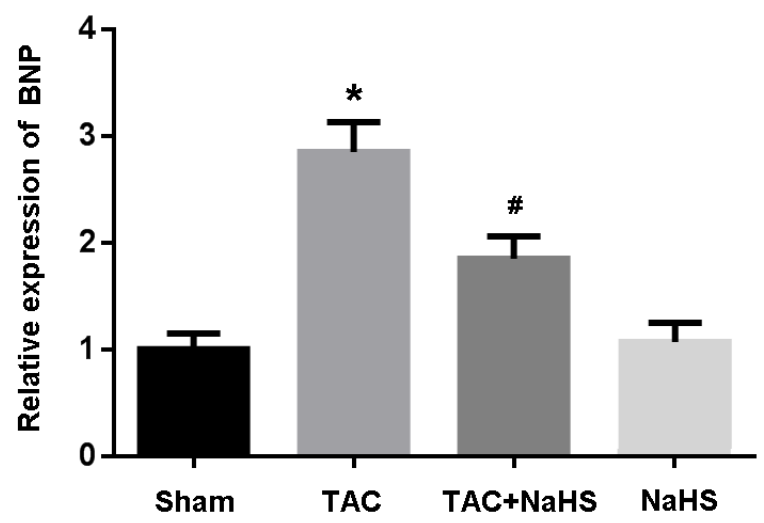

C

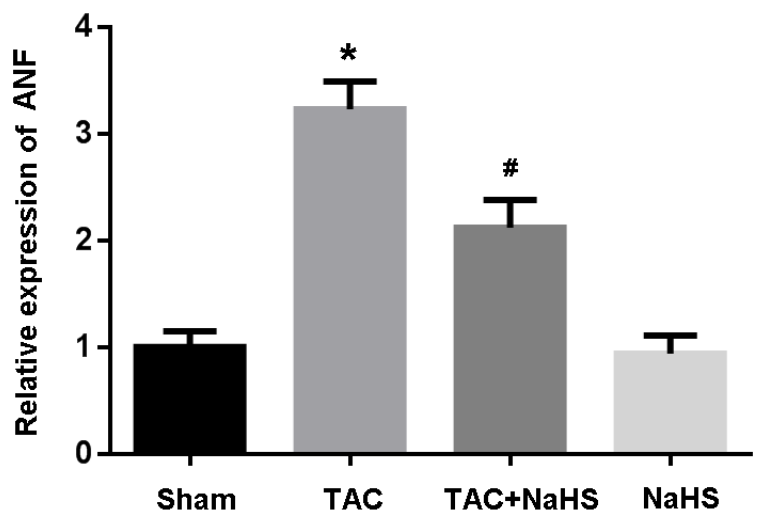

E

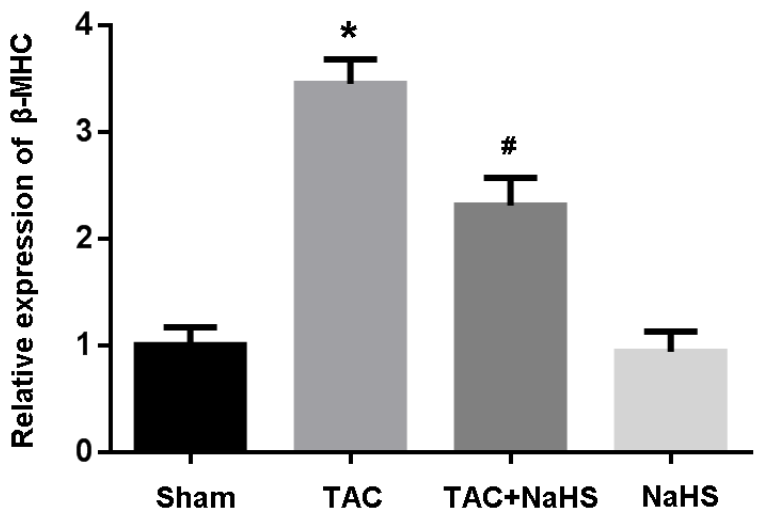

Figure 3: A. Representative images of left ventricular tissue sections stained with hematoxylin-eosin; B. Quantitative analysis of myocyte cross-sectional area; C.-E. Relative expression of ANF, BNP and $\beta$-MHC detected by real-time PCR. ${ }^{*} P<0.05$, vs. Sham; ${ }^{\#} P<0.05$, vs. $\operatorname{TAC}(n=5)$. 
A

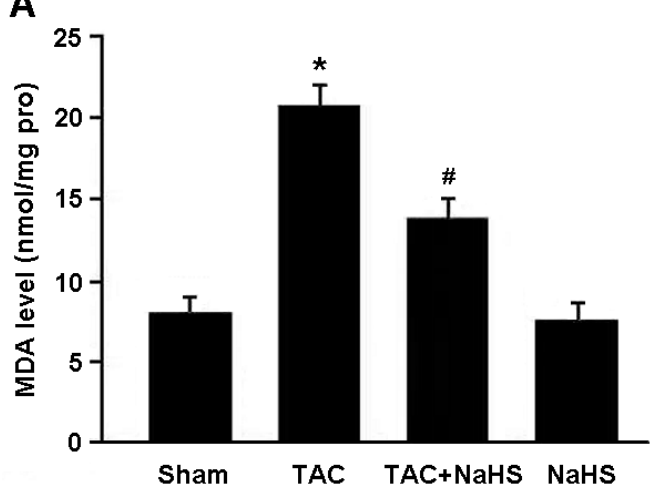

C

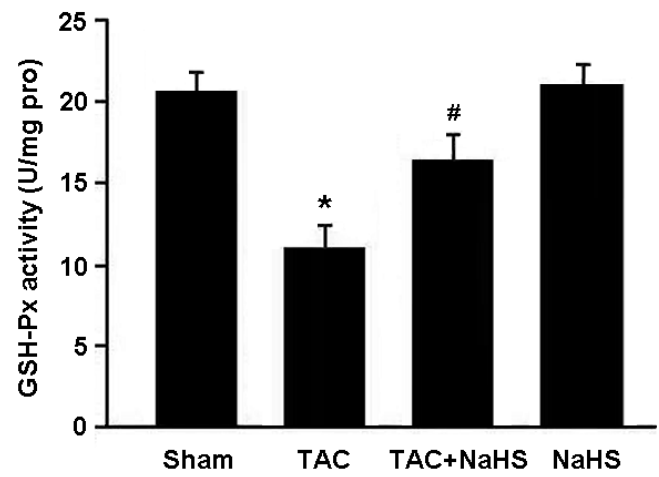

B

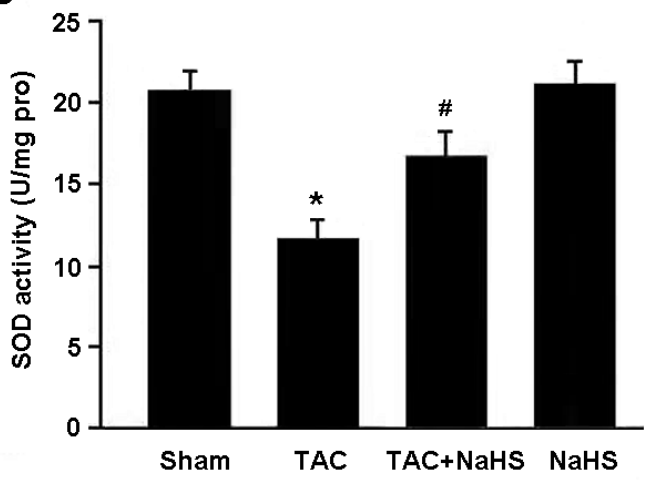

D

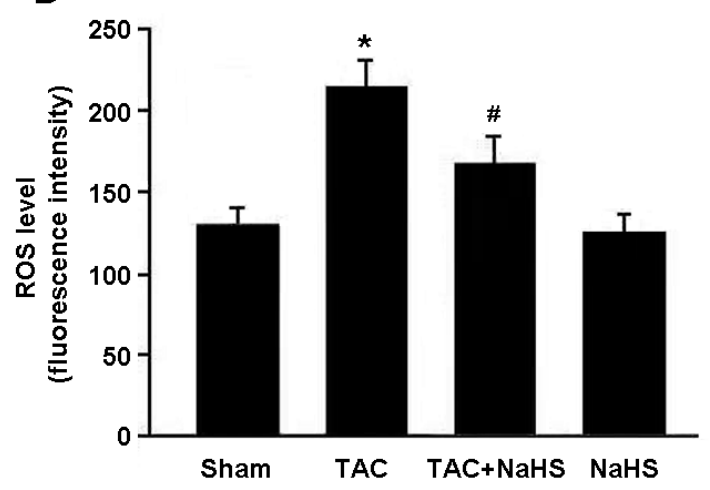

Figure 4: Assessment of oxidative stress in myocardial tissue. A. Malondialdehyde (MDA) level; B. Superoxide dismutase (SOD) activity; C. Glutathione peroxidase (GSH-Px) activity; D. Reactive oxygen species (ROS) level. " $P<0.05$, vs. Sham; ${ }^{~} P<0.05$, vs. TAC $(n=5)$.
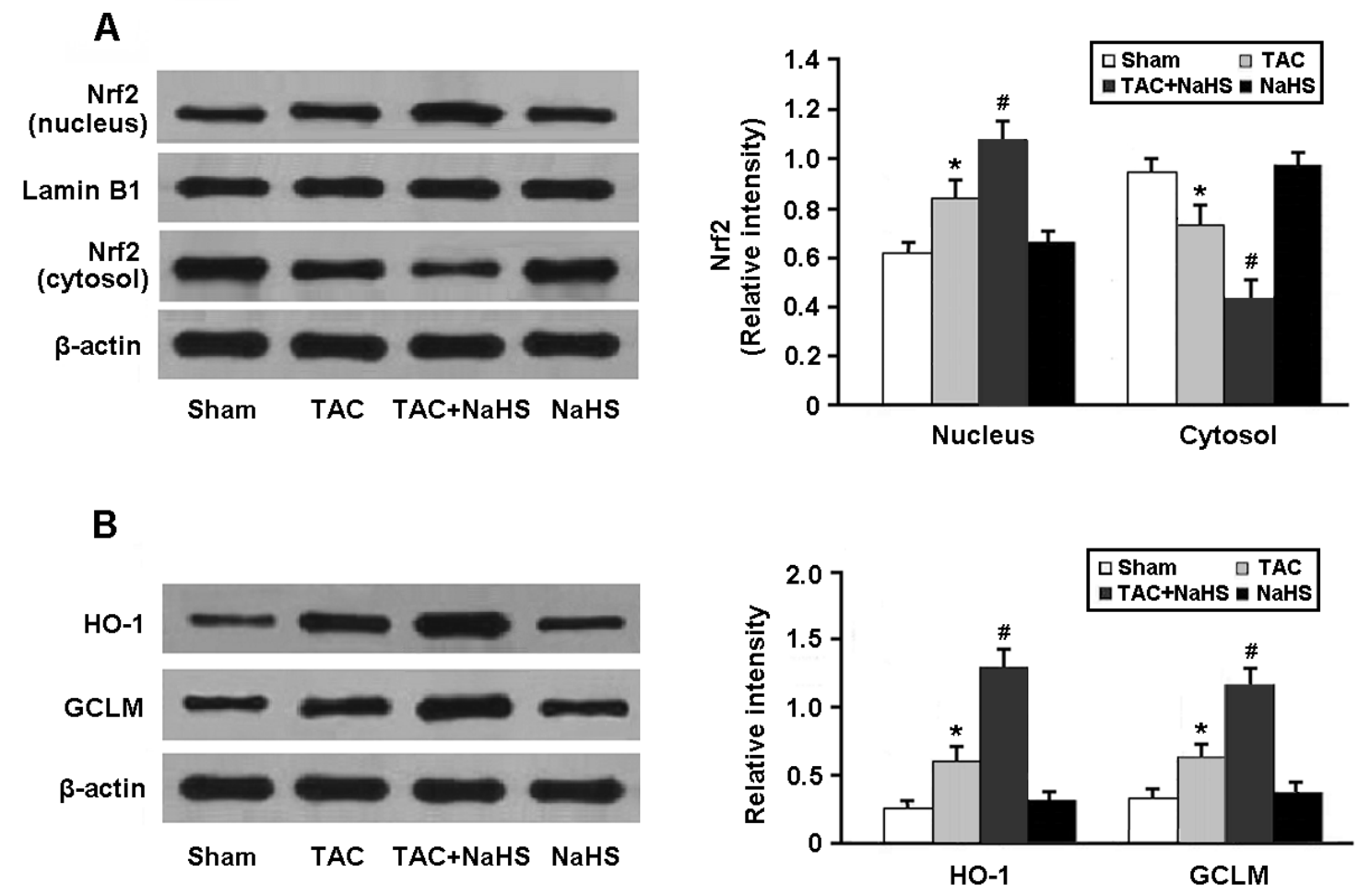

Figure 5: Western blotting analysis of Nrf2 in the nucleus and cytosol (A) and its downstream targets HO-1 and GCLM (B). ${ }^{*} P<0.05$, vs. Sham; ${ }^{\#} P<0.05$, vs. TAC $(n=5)$. 
PI3K is a lipid kinase and generates $\mathrm{PI}(3,4,5) \mathrm{P} 3$, which is a second messenger critical for the translocation of Akt to the cytoplasmic membrane. The activated Akt kinase plays crucial roles in various cellular functions such as cellular survival and proliferation by phosphorylating a number of substrates, including Ikappa B kinase, Bad, caspase-9 and forkhead transcription factors [13]. In the present study, our results suggested that PI3K/

\section{A}
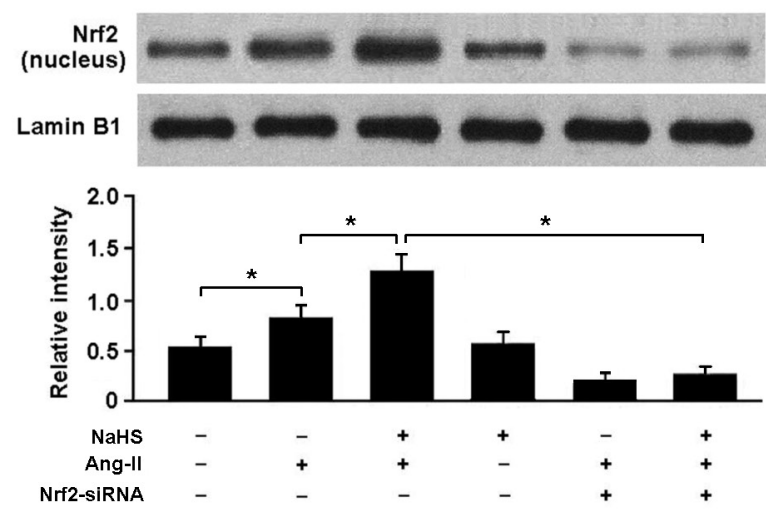

B

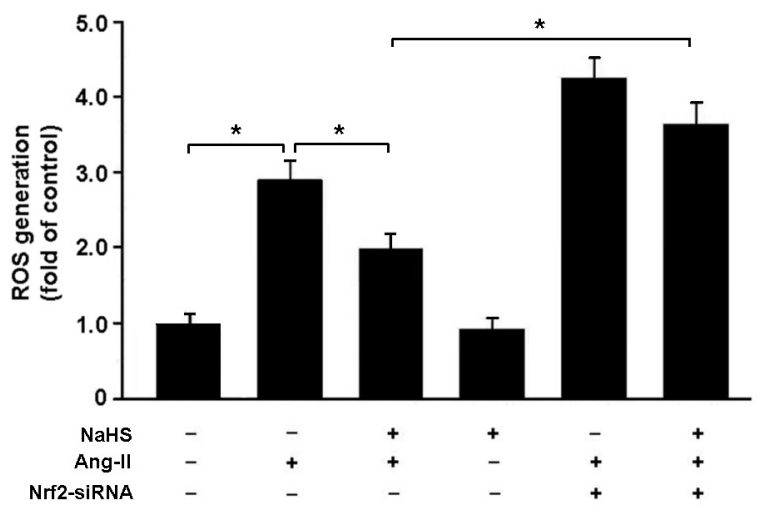

Figure 6: A. Western blotting analysis of Nrf2 nuclear protein in cardiomyocytes. B. Nrf2 siRNA-transfected cells pretreated with NaHS $(100 \mu \mathrm{M})$ for $30 \mathrm{~min}$ prior to exposure to Ang-II $(150 \mathrm{nM})$ for $24 \mathrm{~h}$ exhibited increased ROS production. ${ }^{*} P<0.05(n=5$ independent experiments).

A

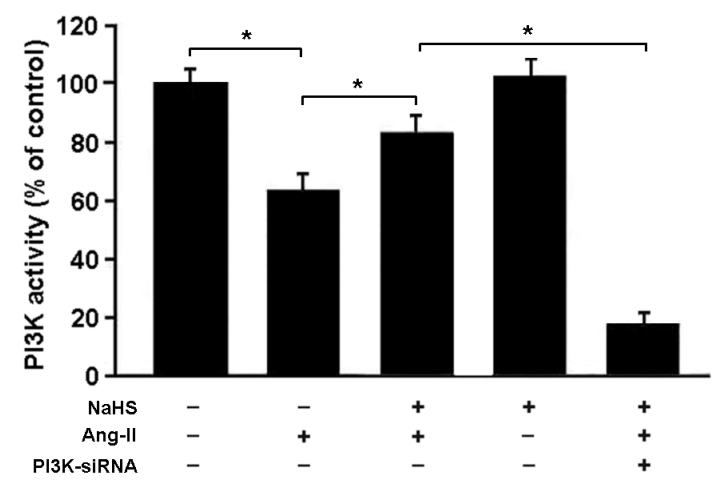

C

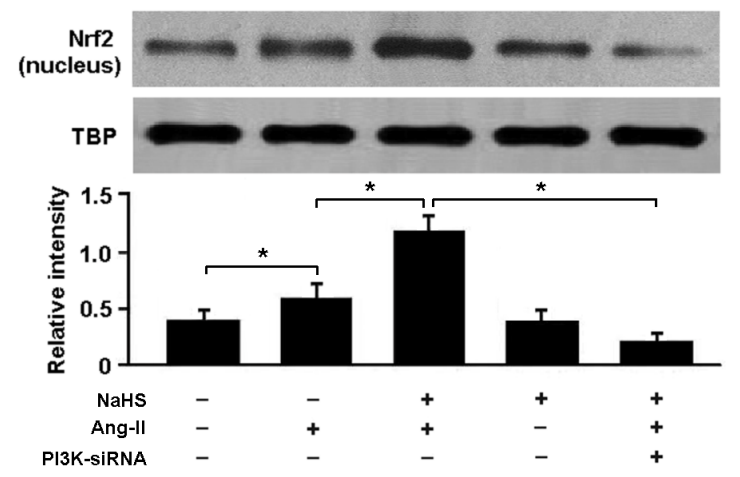

B

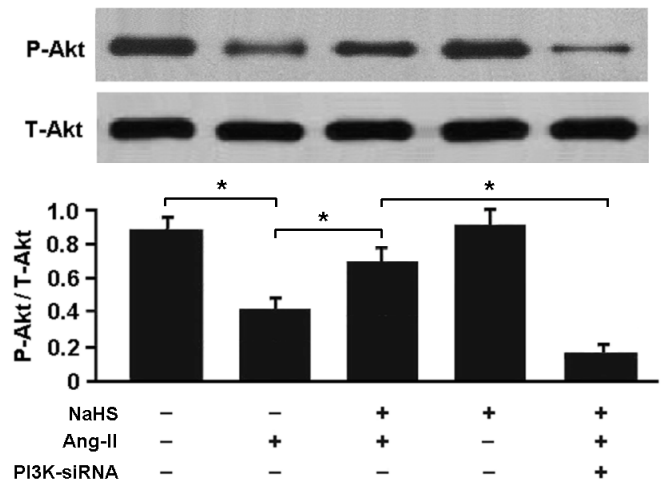

D

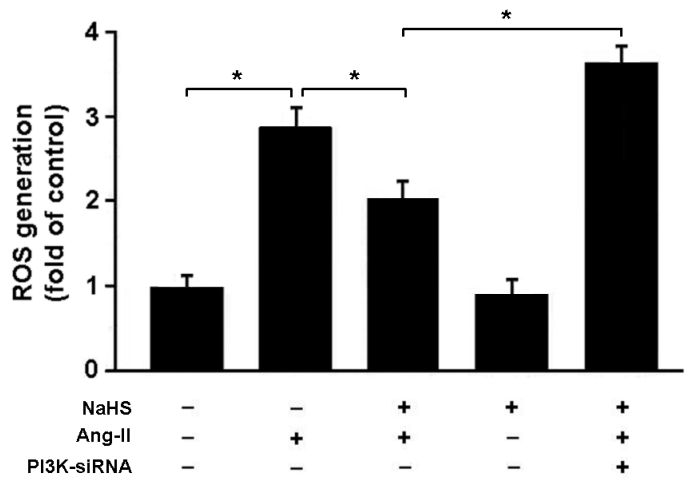

Figure 7: A. PI3K activity in cardiomyocytes was detected by enzyme-linked immunosorbent assay. B. Western blotting analysis of Akt phosphorylation in cardiomyocytes. C. The nuclear protein of Nrf2 were significantly upregulated in cells treated with Ang-II and NaHS and downregulated in PI3K siRNA-transfected cells exposed to Ang-II and NaHS. D. PI3K siRNA-transfected cells pretreated with NaHS $(100 \mu \mathrm{M})$ for $30 \mathrm{~min}$ prior to exposure to Ang-II $(150 \mathrm{nM})$ for $24 \mathrm{~h}$ exhibited increased ROS production. ${ }^{*} P<0.05(n=5$ independent experiments). 
Akt signaling was suppressed in the myocardium of hypertrophic mice, while exogenous administration of NaHS was found to activate PI3K/Akt pathway.

To verify whether $\mathrm{H}_{2} \mathrm{~S}$ regulates $\mathrm{Nrf2}$ via $\mathrm{PI} 3 \mathrm{~K} /$ Akt-dependent pathway, we transfected cardiomyocytes with PI3K siRNA and then subjected them to AngII and NaHS. Our results revealed that suppression of PI3K/Akt signaling was associated with reduced nuclear accumulation of Nrf2 and elevated ROS production in cardiomyocytes treated with NaHS and Ang-II, which suggests that $\mathrm{H}_{2} \mathrm{~S}$ could attenuate oxidative stress via PI3K/Akt-dependent activation of Nrf2 pathway.

In summary, our study reveals that $\mathrm{H}_{2} \mathrm{~S}$ protects against TAC-induced cardiac hypertrophy by attenuating oxidative stress. The antioxidant roles of $\mathrm{H}_{2} \mathrm{~S}$ in myocardial hypertrophy probably depend on the activation of PI3K/Akt pathway, which consequently increases Nrf2 activity and HO-1 and GCLM expression.

\section{MATERIALS AND METHODS}

\section{Animal model}

All experiments were carried out in accordance with the Guide for the Care and Use of Laboratory Animals. The TAC surgery was performed on 8 weeks old male C57BL/6 mice as previously described [14]. The sham group underwent a sham operation involving thoracotomy and aortic dissection without constriction of the aorta. The mice were divided into 4 groups: Sham group, TAC group, TAC+NaHS group (TAC mice were intraperitoneally administered with NaHS solution at a dose of $15 \mu \mathrm{mol} / \mathrm{kg} /$ day) and $\mathrm{NaHS}$ group (sham mice were intraperitoneally administered with NaHS solution). After 4 weeks, animals were sacrificed by cervical dislocation, and the hearts were harvested for analysis.

\section{Cardiomyocyte culture}

Neonatal ventricular myocytes were isolated from 1-2 days old mice. Briefly, myocardial tissue was surgically removed and then dispersed in a series of incubations at $37^{\circ} \mathrm{C}$ in D-Hanks buffered solution containing $1.2 \mathrm{mg} /$ $\mathrm{mL}$ pancreatin and $0.14 \mathrm{mg} / \mathrm{mL}$ collagenase (GIBCO, USA). After centrifugation, the cells were suspended in Dulbecco's modified Eagle medium/F-12 (GIBCO, USA) containing $10 \%$ heat-inactivated foetal bovine serum, 100 $\mathrm{U} / \mathrm{ml}$ penicillin, $100 \mu \mathrm{g} / \mathrm{ml}$ streptomycin, and $0.1 \mathrm{mM}$ bromodeoxyuridine. The dissociated cells were preplated at $37^{\circ} \mathrm{C}$ for $1 \mathrm{~h}$ to separate cardiomyocytes by adherence of cardiac fibroblasts. The cardiomyocytes were then collected and diluted to $1 \times 10^{6}$ cells $/ \mathrm{ml}$ and plated in $1 \%$ gelatin-coated culture dishes.

\section{Measurement of $\mathrm{H} 2 \mathrm{~S}$ content}

The $\mathrm{H}_{2} \mathrm{~S}$ levels in myocardial tissue were measured by the methylene blue method which was described by Zhou et al. [15]. This method is based on the reaction of sulfide with N,N-dimethyl-p-phenylenediamine, in a ferric chloride catalyzed reaction with a 1:2 stoichiometric ratio to give the methylene blue dye, which is detected by spectrophotometry.

\section{Echocardiographic study}

Cardiac hypertrophy was evaluated by echocardiographic analysis of heart size, including left ventricular posterior wall thickness at end-systole (LVPWs), left ventricular posterior wall thickness at enddiastole (LVPWd), interventricular septum thickness at end-diastole (IVSd) and interventricular septum thickness at end-systole (IVSs). All measurements were averaged for 3 consecutive cardiac cycles.

\section{Histological analysis}

Left ventricular tissue was fixed in $10 \%$ buffered formalin, embedded in paraffin, and sliced into 5 - $\mu$ m-thick sections. Slides were stained with hematoxylin-eosin (HE) and observed under a light microscope. Myocyte crosssectional area (CSA) was measured using Pro Plus 6.0 image analysis software.

\section{Measurement of oxidative stress}

Oxidative stress was assessed by detecting malondialdehyde (MDA) levels, superoxide dismutase (SOD) and glutathione peroxidase (GSH-Px) activities and ROS generation in the myocardial tissue according to the instructions of detection kits (Jiancheng Biotech, China).

\section{siRNA transfection}

Cardiomyocytes were seeded into 6-well plates for $24 \mathrm{~h}$ and then transfected with NF-E2-related factor 2 (Nrf2) or PI3-kinase (PI3K) siRNA using Lipofectamine 2000 reagents. Subsequently, the cells were treated with angiotensin II (Ang-II) for $30 \mathrm{~min}$ after incubated with or without NaHS $(100 \mu \mathrm{M})$.

\section{PI3K activity assay}

PI3K activity was determined using an enzymelinked immunosorbent assay (ELISA) kit (Echelon Biosciences, Salt Lake City, USA) following the manufacturer's instructions. In this method, PI3K activity 
was evaluated by detecting the conversion of $\mathrm{PI}(4,5) \mathrm{P} 2$ into $\mathrm{PI}(3,4,5) \mathrm{P} 3$.

\section{Real-time PCR}

Total RNA was isolated from myocardial tissue using TRIzol reagent (Invitrogen, USA). RNA was reverse transcribed using SuperScript First-Strand cDNA System (Invitrogen, USA). Real-time reaction was run and analyzed using a real-Time PCR system (ABI 7300). The primer sequences used in this study were as follows: ANF, 5'-CTCCGATAGATCTGCCCTCTTGAA-3' and 5'-GGTACCGGAAGCTGTTGCAGCCTA-3'; BNP, 5'-GCTCTTGAAGGACCAAGGCCTCAC-3' and 5'-GATCCGATCCGGTCTATCTTGTGC-3'; $\beta$-MHC, 5'-CAGACATAGAGACCTACCTTC-3' and 5'-CAGCATGTCTAGAAGCTCAGG-3'. The relative expression of mRNA was calculated using the comparative cycle threshold $(\mathrm{CT})\left(2^{-\Delta \Delta \mathrm{CT}}\right)$ method.

\section{Western blotting}

The samples were homogenized in $0.1 \%$ SDS buffer and the lysates were centrifuged at $12,000 \mathrm{rpm}$ for $15 \mathrm{~min}$. The supernatant was collected and the protein concentration was determined. The extracted protein was separated on SDS-PAGE gel and transferred onto PVDF membrane (Millipore, USA). The membrane was blocked with $5 \%$ bovine serum albumin for $1 \mathrm{~h}$ to reduce non-specific binding. Then, the blot was incubated with the primary antibody for $12 \mathrm{~h}$ at $4^{\circ} \mathrm{C}$. The antibodies used in this study were purchased from Cell Signaling Technology. After washing, the blot was incubated with HRP-conjugated secondary antibody (Santa Cruz, USA) for $1 \mathrm{~h}$ at room temperature. Finally, the signal was detected using the enhanced chemiluminescence kit (Amersham Biosciences).

\section{Statistical analysis}

In this study, statistical analysis was performed using SPSS software. The differences between two groups were determined by Student's t-test, and the differences among more than two groups were determined by Analysis of Variance followed by SNK-q method. $P<0.05$ was considered statistically significant.

\section{ACKNOWLEDGMENTS}

This study was supported by the Found of Tianjin Health Bureau (2014KR02 to C.J.Z), Chinese Postdoctoral Science Foundation (2012M0585 to C.J.Z), Jiangsu Haosen pharmaceutical Limited by Share Ltd (2016-Young scholar support project to C.J.Z), Hainan
Liou pharmaceutical Limited by Share Ltd (2016-Young scholar support project to C.J.Z), Xuzhou Enhua pharmaceutical Limited by Share Ltd (2016-Young scholar support project to C.J.Z), and Shanghai Zhongxi pharmaceutical Limited by Share Ltd (2016-Young scholar support project to C.J.Z).

\section{CONFLICTS OF INTEREST}

The authors declare no potential conflicts of interest.

\section{REFERENCES}

1. Lyon RC, Zanella F, Omens JH, Sheikh F. Mechanotransduction in cardiac hypertrophy and failure. Circ Res. 2015; 116:1462-1476.

2. Maillet M, van Berlo JH, Molkentin JD. Molecular basis of physiological heart growth: fundamental concepts and new players. Nat Rev Mol Cell Biol. 2013; 14:38-48.

3. Shimizu I, Minamino T. Physiological and pathological cardiac hypertrophy. J Mol Cell Cardiol. 2016; 97:245-262.

4. Szabó C. Hydrogen sulphide and its therapeutic potential. Nat Rev Drug Discov. 2007; 6:917-935.

5. Pan LL, Liu XH, Gong QH, Yang HB, Zhu YZ. Role of cystathionine $\gamma$-lyase/hydrogen sulfide pathway in cardiovascular disease: a novel therapeutic strategy? Antioxid Redox Signal. 2012; 17:106-118.

6. Liu YH, Lu M, Hu LF, Wong PT, Webb GD, Bian JS. Hydrogen sulfide in the mammalian cardiovascular system. Antioxid Redox Signal. 2012; 17:141-185.

7. Elrod JW1, Calvert JW, Morrison J, Doeller JE, Kraus DW, Tao L, Jiao X, Scalia R, Kiss L, Szabo C, Kimura $\mathrm{H}$, Chow CW, Lefer DJ. Hydrogen sulfide attenuates myocardial ischemia-reperfusion injury by preservation of mitochondrial function. Proc Natl Acad Sci U S A. 2007; 104:15560-15565.

8. Calvert JW, Elston M, Nicholson CK, Gundewar S, Jha S, Elrod JW, Ramachandran A, Lefer DJ. Genetic and pharmacologic hydrogen sulfide therapy attenuates ischemia-induced heart failure in mice. Circulation. 2010; 122:11-19.

9. Meng G, Zhu J, Xiao Y, Huang Z, Zhang Y, Tang X, Xie L, Chen Y, Shao Y, Ferro A, Wang R, Moore PK, Ji Y. Hydrogen Sulfide Donor GYY4137 Protects against Myocardial Fibrosis. Oxid Med Cell Longev. 2015; 2015:691070.

10. Cave A, Grieve D, Johar S, Zhang M, Shah AM. NADPH oxidase-derived reactive oxygen species in cardiac pathophysiology. Philos Trans R Soc Lond B Biol Sci. 2005; 360:2327-2334.

11. Li JM, Gall NP, Grieve DJ, Chen M, Shah AM. Activation of NADPH oxidase during progression of cardiac hypertrophy to failure. Hypertension. 2002; 40:477-484.

12. Kaspar JW, Niture SK, Jaiswal AK. Nrf2:INrf2 (Keap1) 
signaling in oxidative stress. Free Radic Biol Med. 2009; 47:1304-1309.

13. Manning BD, Cantley LC. AKT/PKB signaling: navigating downstream. Cell. 2007; 129:1261-1274.

14. Liu L, An X, Li Z, Song Y, Li L, Zuo S, Liu N, Yang G, Wang H, Cheng X, Zhang Y, Yang X, Wang J. The H19 long noncoding RNA is a novel negative regulator of cardiomyocyte hypertrophy. Cardiovasc Res. 2016; 111:5665.
15. Zhou X, An G, Lu X. Hydrogen sulfide attenuates the development of diabetic cardiomyopathy. Clin Sci (Lond). 2015; 128:325-335. 